\title{
Beam Coupling to Optical Scale Accelerating Structures
}

\author{
Christopher M.S. Sears ${ }^{1}$, Robert L. Byer ${ }^{2}$, Eric R. Colby ${ }^{1}$, Benjamin M. \\ Cowan ${ }^{1}$, Rasmus Ischebeck ${ }^{1}$, Melissa R. Lincoln ${ }^{1}$, Tomas Plettner ${ }^{2}$, \\ Robert H. Siemann ${ }^{1}$, James E. Spencer ${ }^{1}$ \\ 1 - Stanford Linear Accelerator Center, Menlo Park, CA 94025 \\ 2 - Stanford University, Stanford, CA 94305
}

\begin{abstract}
Current research efforts into structure based laser acceleration of electrons utilize beams from standard RF linacs. These beams must be coupled into very small structures with transverse dimensions comparable to the laser wavelength. To obtain decent transmission, a permanent magnet quadrupole (PMQ) triplet with a focusing gradient of $560 \mathrm{~T} / \mathrm{m}$ is used to focus into the structure. Also of interest is the induced wakefield from the structure, useful for diagnosing potential accelerator structures or as novel radiation sources.
\end{abstract}

PACS: 41.75.Jv, 41.60.Bq, 41.85.Lc

Current RF accelerators typically have accelerating gradients on the order of a few tens of megavolts per meter. For the accelerators under consideration for next generation high energy physics experiments this may be improved to $\sim 70 \mathrm{MeV} / \mathrm{m}$. To obtain the beam energies needed for physics experiments this results in an accelerator 10 's of $\mathrm{km}$ in length. As high energy physics continues to demand ever higher beam energies, civil construction is a growing burden on the overall cost and may one day prevent continued progress with conventional accelerating techniques. In future, physics will require new technologies with greater accelerating gradients to reach high beam energies while reducing machine size. One of the main fields of active research to this end involves the use of lasers instead of RF fields for acceleration.

Laser acceleration in optical scale electromagnetic structures offers the potential of high acceleration gradients $(\geq 1 \mathrm{GeV} / \mathrm{m})$ and good laser to electron energy efficiency [1]. However, as with conventional RF \& microwave structures, most structures for laser acceleration [2,3] have transverse apertures comparable to the accelerating wavelength. For laser acceleration $(\lambda \approx 1 \mu \mathrm{m})$ this presents unique challenges for electron focusing and transmission through the structures. In these proceedings we report on the design, fabrication, and measurement of a permanent magnet quadrupole triplet for focusing the beam through such structures.

Conventional electron optics prove insufficient to obtain the small spot necessary for transmission through an optical structure. The chromatic aberrations for a long focal length triplet prevent reaching small $\beta^{*}$. For optimal transmission through a fiber $\beta^{*}$ must equal half the length of the fiber. For structures being considered for the 
E163 experiment this means $\beta^{*}$ in the range of 0.5 to $2.5 \mathrm{~mm}$. Equation 1 gives the relation for $\beta^{*}$ as a function of the focal length of the triplet.

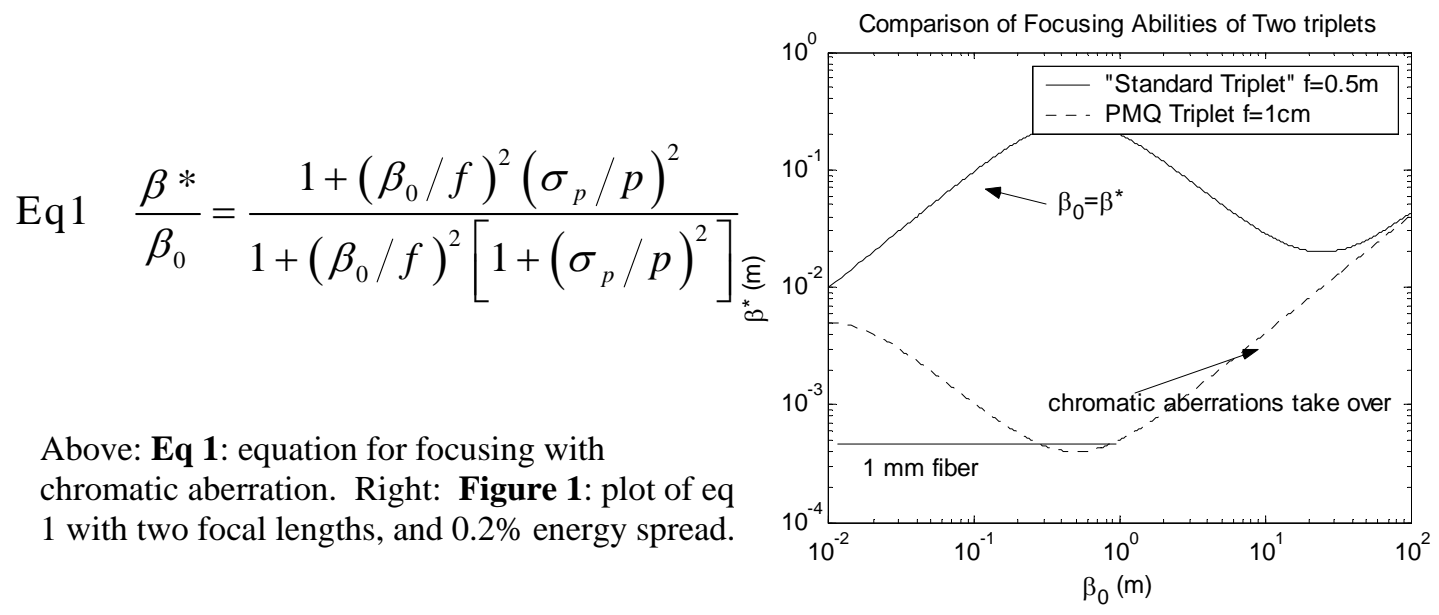

From figure 1 we see that for a standard triplet chromatic effects take over before a sufficiently tight focus is reached. A short focal length permanent magnet quadrupole however can obtain the necessary focusing. The total transmission can then be determined by comparing the transmitted emittance of the structure to the initial emittance. The transmitted emittance is determined by the allowable range of position and angles that can pass through the structure, as illustrated in figure 2. For a $1 \mathrm{~mm}$ long fiber with a $5 \mu \mathrm{m}$ aperture the transmitted emittance is $0.03 \mathrm{~mm}$-mrad (geometric). This compares to an expected geometric emittance of $0.03 \mathrm{~mm}$-mrad RMS at E163, giving a transmission of $~ 15 \%$.
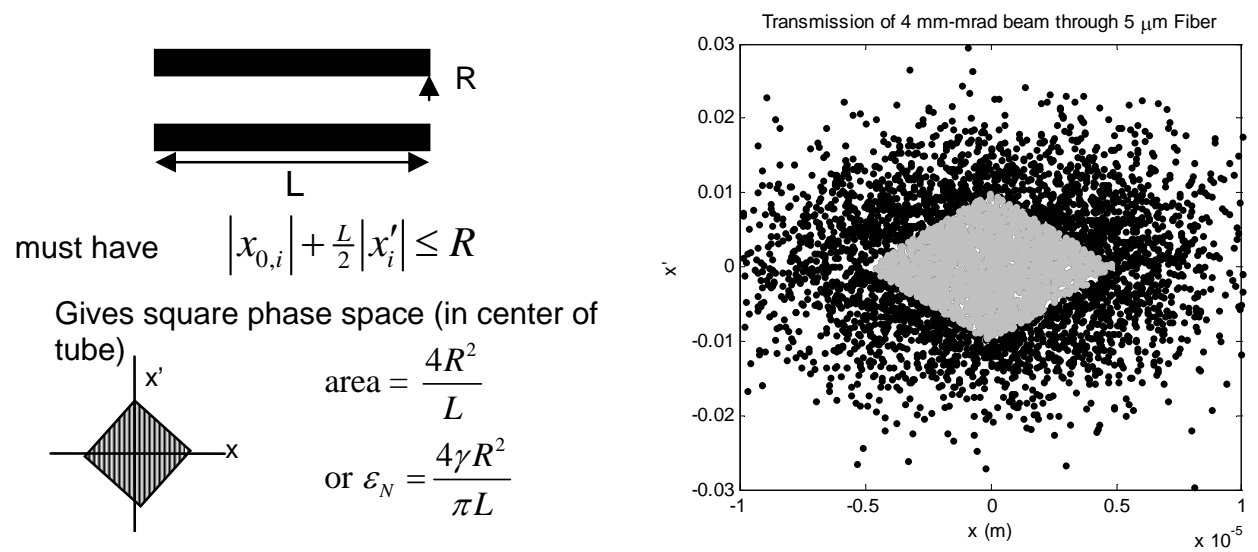

Figure 2 Transmission through small apertures. Right: PIC simulation of phase space filtering due to aperture. $\sim 15 \%$ of particles pass through. 


\section{Design of the PMQ Triplet}

The PMQ triplet was designed using the Radia [4] magnetostatic simulation code. The triplet is similar in spirit to the device designed by Jae Lim [5] at UCLA, though with two significant design differences. First, instead of the original iron-free arrangement proposed by Halbach [6] and comprised of 16 magnet blocks having 5 unique polarization orientations relative to the block shape, this design uses four magnet blocks with four iron pole tips and just one polarization orientation. This greatly simplifies fabrication, and also gives higher fields for a given aperture size. The second difference is that the triplet, like all the E163 hardware, is designed to sit entirely in vacuum. This allows for greater flexibility in swapping out experiment components, makes the total distance between components smaller, and simplifies issues like electron-laser alignment and diagnostic insertions.

The quads sit atop a common mount, with the two upstream quads driven by stepper motors for tuning. Tuning with the quad gaps allows the triplet to accommodate a range of beam energies and also can correct for an initial beam asymmetry or differences in quad strength due to fabrication error. A third motor moves the stage as a whole to locate the focal point. Figure 3 shows a picture of the entire triplet and a CAD image from RADIA of one quadrupole. The aperture is $3 \mathrm{~mm}$

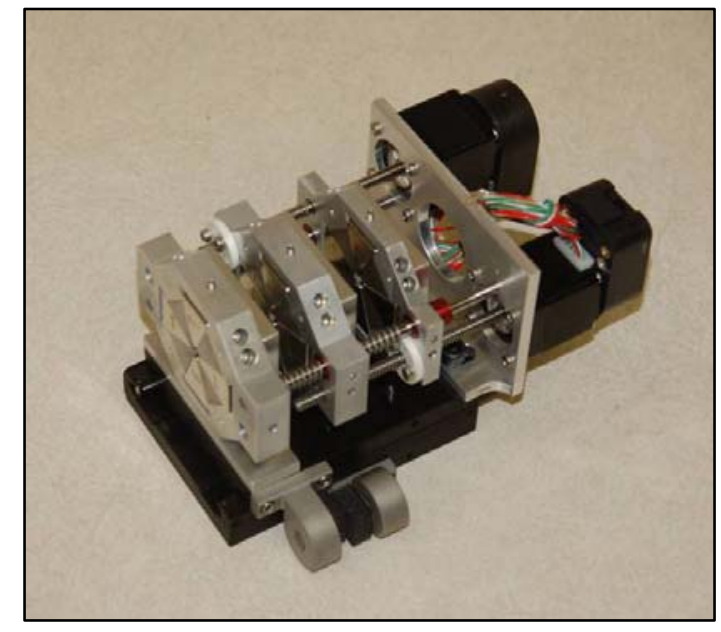

Figure 3 Top Left: Picture of completed triplet assembly including motors and stage. The entire assembly measures $\sim 8 \times 8 \times 12 \mathrm{~cm}$. Top Right: CAD image from RADIA of a signal PMQ showing the magnet polarization directions. Bottom Right: Focusing ability of entire triplet as a function of the initial spot size. This plot includes effects due to higher order multipoles due to fabrication errors (see next section).
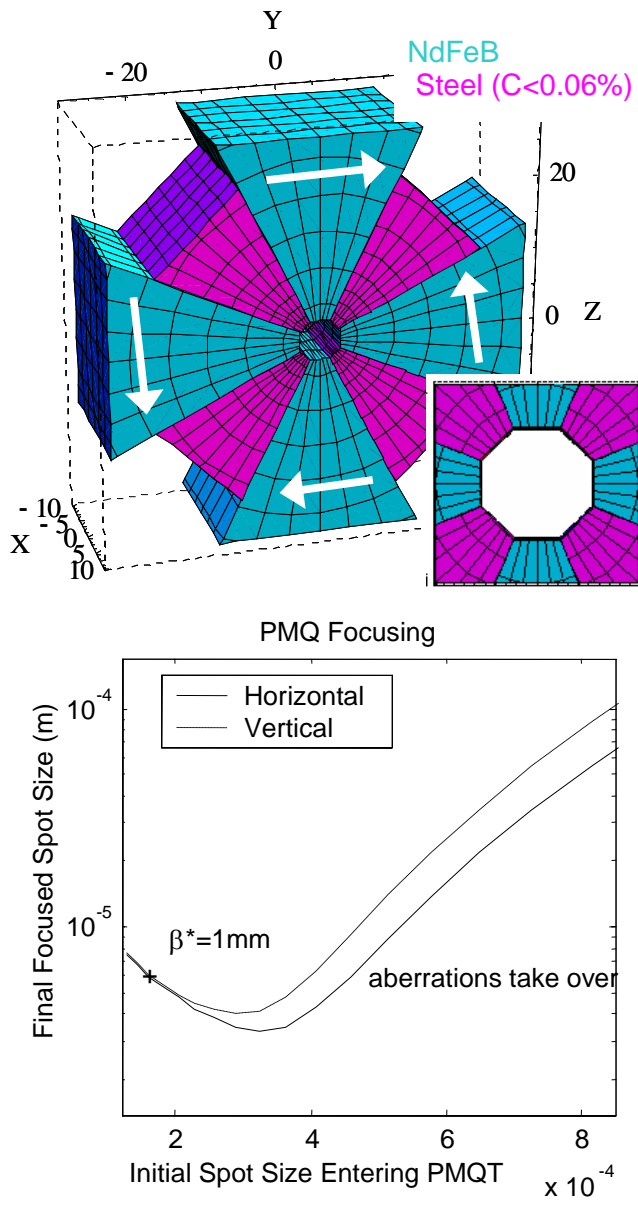
in radius. The magnet blocks are $20 \mathrm{~mm}$ high, the iron poles $16 \mathrm{~mm}$. The pole tips are flat to simplify fabrication. The triplet consists of one thinner quadrupole at $7 \mathrm{~mm}$ and two thicker quadrupoles, $13 \mathrm{~mm}$ each. The plot on the right of figure 3 shows the expected focusing ability of the triplet. A cross mark shows the planned $\beta^{*}$ for a $1 \mathrm{~mm}$ long fiber. The plot includes the effect of the measured field errors. Although worse than the chromatic effects, the field errors are still sufficiently small to allow the triplet to focus sufficiently. The triplet was fabricated in house at SLAC.

\section{Measuring the PMQ Triplet}

After fabrication, the PMQ triplet was thoroughly measured to verify the RADIA simulations and determine any field errors due to fabrication. The triplet was measured via 3 techniques: a standard hall probe, a pulsed wire [7], and a rotating coil. Each method had its own benefits and drawbacks. The hall probe was used to find the effective quadrupole gradient of each quad: $450 \mathrm{~T} / \mathrm{m}$ for the smaller quad and $560 \mathrm{~T} / \mathrm{m}$ for the larger two. While the hall probe could in theory be used to obtain all useful field measurements, the small aperture of the triplet and relatively large size of the hall probe resulted in significant interference between the pole tips and probe holder. The slow speed of the hall probe also made data collection tedious for more than a few scans.

To obtain information on the relative alignment between the three quadrupoles we turned to a pulsed wire technique. Figure 4 shows a schematic and sample scan from a pulsed wire measurement. In this particular setup an oscilloscope is used to read out the full longitudinal profile of a scan. The pulsing technique produces a wave on the string whose amplitude is related to the first integral of the magnetic field. An RC differentiator before the scope gives back the original field. Placed along side the triplet is a simple dipole with a well known field for calibrating the scope traces. From the pulse wire measurements we were able to determine the relative centers of
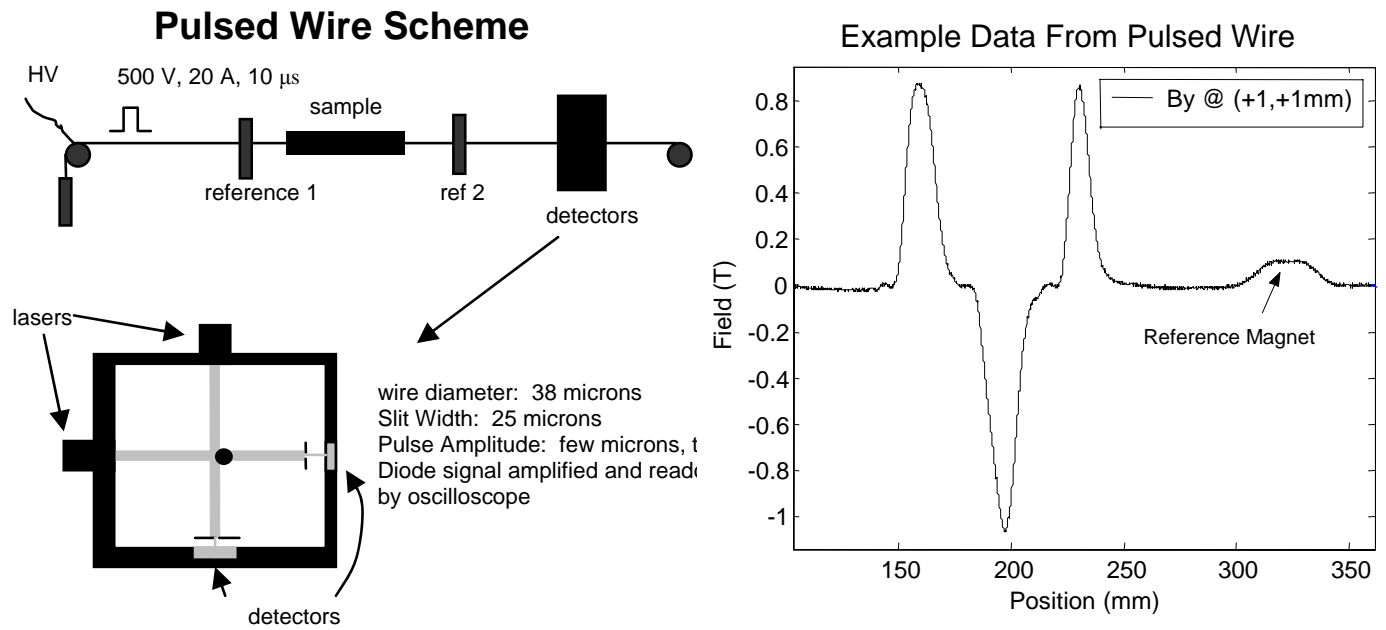

Figure 4 Left: schematic of pulsed wire setup. Right: Two scans of $B_{y}$ through triplet and a reference magnet. 


\begin{tabular}{|c|c|}
\hline \begin{tabular}{|l} 
Center Quad \\
Misalignment
\end{tabular} & \\
\hline Horizontal & $-0.043 \mathrm{~mm}$ \\
\hline Vertical & $0.097 \mathrm{~mm}$ \\
\hline kew Error & $<2 \mathrm{mrad}$ \\
\hline
\end{tabular}

the three quads, as well as check for a skew error on the three (table 1). The values found are well within limits set by simulation, giving negligible emittance increase. The pulsed wire technique has two significant drawbacks. The first is dispersion of the acoustic waves on the string. This problem is manifest on the scope trace as small oscillations after sharp transitions in the magnetic field. This can, however, be corrected for mathematically by applying an appropriate phase shift to the Fourier spectrum of the scope trace and then transforming back to get the corrected scan. A more difficult problem, at least in our setup, was non-linearity in the detection scheme. The acoustic wave is readout by observing the shadow in a laser beam cast by the wire on a narrow slit. However, because of diffraction, the shadow has a Bessel function shape. This could be corrected in later versions of the setup by imaging the wire onto the slit.

The final measurement needed on the PMQ triplet was to quantify the field quality. While beam transport simulations show the beam focusing is relatively insensitive to higher multipoles; the focusing aberrations are dominated by chromatic effects; strong enough multipole errors could hinder focusing. To check the multipole content of the quads a rotating coil scan was performed. A custom coil was fabricated with a $1.5 \mathrm{mmx} 1 \mathrm{~mm}$ area and 15 turns. Figure 5 shows a picture of the coil in front of one of the PMQs and an example data scan. The coils scans revealed a surprising decapole term, the source of which has yet to be determined. Simulations predict only a small dodecapole term. There are also small sextupole, octopole, and dodecapole terms. While the decapole is relatively large, beam transport simulations show the triplet will still focus the beam as needed for coupling to optical structures.
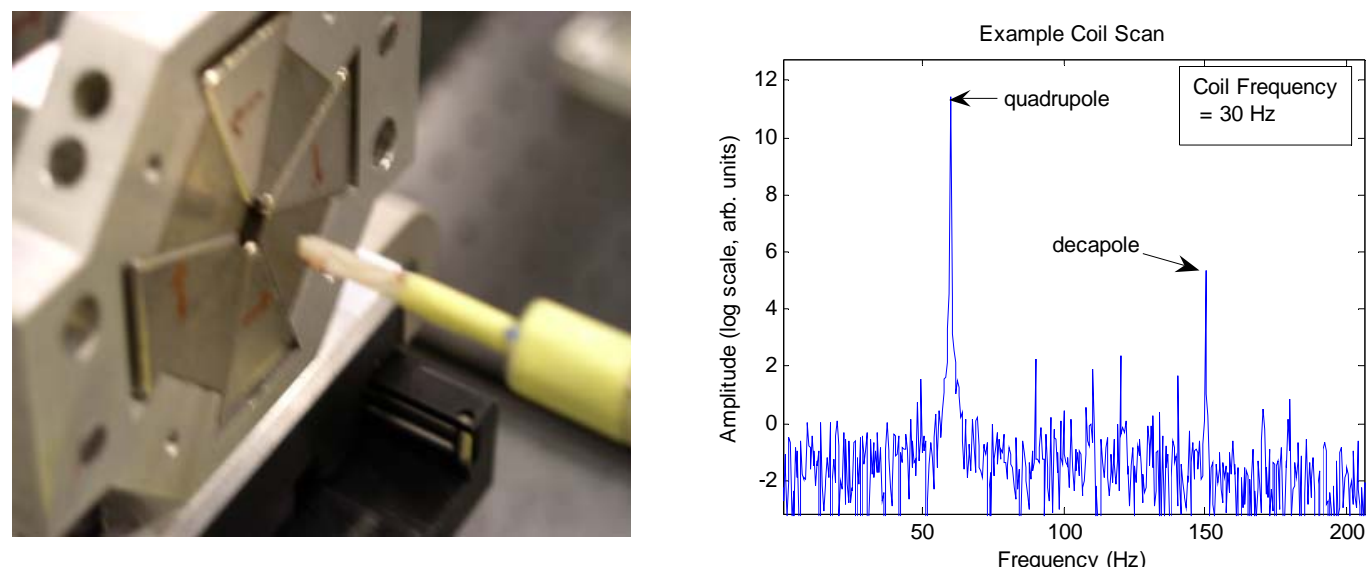

Figure 5 Left: Image of coil near quadrupole aperture. Right: Example data from a coil scan. Measurements found a large decapole term, not expected from simulation. 


\section{Resonant Wakefield from an Optical Structure}

Prior to actual acceleration experiments in which a laser and electron beam will be simultaneously coupled to the structure, a set of experiments using only the structure and PMQ triplet are planned to determine the frequencies at which the structure has speed of light modes. This will be done by observing the wakefield from the structure with a spectrometer. A simulation of a fiber bragg structure [8] shows the formation of a speed-of-light phase velocity wake (figure 6). The total energy radiated from a 50 $\mathrm{pC}$ bunch is $\sim 0.1 \mathrm{~nJ}$ with an additional $2 \mathrm{~nJ}$ of non-resonant Cherenkov radiation leaking out the sides of the fiber. The actual fiber that will be used in the experiment is a commerically available photonic bandgap fiber [9]. Efforts are on going to simulate the wakefield from this fiber.
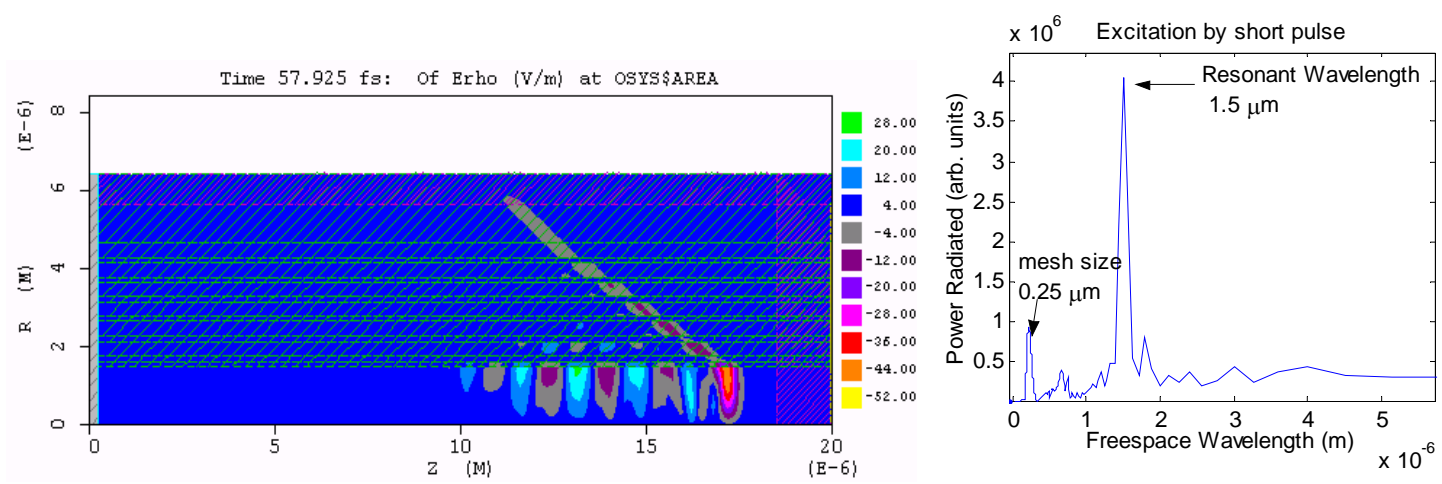

Figure 6 Simulation of resonant wake in an bragg fiber. Left is a snapshot of wake from a single point charge on axis. Right is the fft of the power through the end of the fiber showing both the main excitation of the fiber as well as a peak due to numerical dispersion on the mesh.

\section{Conclusion}

We have designed, fabricated, and measured a permanent magnet quadrupole triplet for coupling an electron beam into small structures for laser acceleration experiments. While a significant decapole term was found in coil measurements, simulation shows the triplet will still perform as needed. The PMQ triplet will allow for a set of experiments testing novel structures with hopes of obtaining acceleration gradients in excess of $1 \mathrm{GeV} / \mathrm{m}$. Initial experiments will use the triplet to focus the beam and observe resonant Cherenkov wake in structures without the laser in order to determine the precise frequency of the accelerating mode.

\section{ACKNOWLEDGMENTS}

We would like to thank Jae Lim and James Rosenzweig for discussions of PMQ design, fabrication, and measurement. We would also like to thank Zach Wolf and rest of the SLAC magnetic measurements group for help with the PMQ triplet field measurements. This work is supported by Department of Energy contracts DEAC02-76SF00515 and DE-FG02-03ER41276. 


\section{References}

1. Y.C. Neil Na and R. H. Siemann, Phys. Rev. ST Accel. Beams 8, 072401 (2005)

2. Lin, X. E., Phys. Rev. ST Accel. Beams, 4, 051301 (2001).

3. B. Cowan, Phys. Rev. ST Accel. Beams 6, 101301 (2003)

4. PAC97 Conference, Vancouver, May 1997: P. Elleaume, O. Chubar and J. Chavanne

5. J. Lim et al. Phys. Rev. ST Accel. Beams 8, 072401 (2005)

6. K. Halbach, NIM 169 (1980)

7. Warren, R.W. NIM A, A272 (1988) 257-263

8. Mizrahi and L. Schächter, Phys. Rev. E 70, 016505 (2004).

9. Thorlabs HC-633-01. See http://www.thorlabs.com/thorcat/14000/14015-M01.pdf 